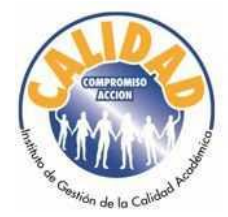

Vicerrectoría Académica

Instituto de Gestión de la Calidad Académica

Co-creando Excelencia

http://revistas.uned.ac.cr./index.php/revistacalidad

Сагге electrónica: revistacalidad国uned.ac.сг

\title{
La internacionalización en el aula en Licenciaturas en Educación Infantil en el Caribe colombiano: una perspectiva docente
}

\begin{abstract}
Internationalization in the learning environments in Early Childhood Education Degrees in the Colombian Caribbean: a teaching perspective
\end{abstract}

Luisa F. Echeverría King ${ }^{1}$

lecheverriaking@gmail.com

Servicio Nacional de Aprendizaje (SENA),

Colombia

(10https://orcid.org/0000-0002-5172-1487
Fuensanta Hernández-Pina²

fhpina@me.com

Universidad de Murcia, España

(1) https://orcid.org/0000-0003-2339-8149

Diana Lago de Vergara ${ }^{3}$

dianalago20@yahoo.es

Universidad de Cartagena, Colombia

(iD) https://orcid.org/0000-0001-8207-7797

DOI: $h$ ttp://dx.doi.org/10.22458/caes.v11i2.2831

Volumen 11, Número 2

30 de noviembre de 2020

pp. $51-79$

Recibido: 02 de marzo del 2020

Aprobado: 12 de octubre del 2020

\footnotetext{
${ }^{1}$ Asesora de Internacionalización del Servicio Nacional de Aprendizaje (SENA) en Colombia y Consultora de Educación Superior. Estudiante del Doctorado en Educación de la Universidad de Murcia, España. Email: lecheverriaking@gmail.com.

${ }^{2}$ Catedrática Emérita de Métodos de Investigación y Diagnóstico en Educación, en la Universidad de Murcia. Doctora por la Universidad de Valencia desde 1977. Email: fhpina@me.com.

${ }^{3}$ Doctora en Filosofía y Ciencias de la Educación, Universidad Complutense de Madrid, España. Magister en Proyectos de Desarrollo Social, Universidad del Norte, Barranquilla. Profesora Titular, Universidad de Cartagena. Email: dianalago20@yahoo.es.
}

La internacionalización en el aula en Licenciaturas en Educación Infantil en el Caribe colombiana: una perspectiva dacente

Luisa F. Echeverría King, Fuensanta Hernández-Pinal, Diana Laga de Vergara

DII: http://dx.dai.org/l0.22458/caes.vlli2.2831

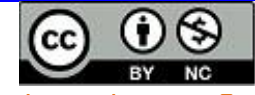

Artícula protegida par licencia Creative Cammans 


\title{
Resumen
}

La internacionalización es un proceso que apoya al desarrollo de la calidad de las instituciones. En los programas de Licenciatura en Educación Infantil, los cuales forman al profesorado de las próximas generaciones, la internacionalización juega cada vez un rol más importante pues la globalización y la sociedad del conocimiento ha tensionado la labor docente. Según esta idea, el presente artículo muestra los resultados de uno de los objetivos de una tesis doctoral y se concentra en comprender cómo se desarrolla la internacionalización en el aula en programas de Licenciatura en Educación Infantil en las instituciones objeto de estudio, Corporación Universitaria del Caribe y la Universidad de la Guajira en Colombia. Este análisis se elaboró con los lineamientos para el aseguramiento de la calidad de programas en Colombia y la Guía para la Calidad en la Internacionalización propuesta por el Consejo Europeo de Agencias de Acreditación. Por medio de 17 entrevistas a docentes, se recogieron los datos. Se observa que en cuanto a metodologías docentes, se priorizan pedagogías activas, aprendizaje colaborativo en línea con pares internacionales, así como debates y aprendizaje basado en problemas. Para evaluar se usan proyectos, talleres, presentaciones y exámenes escritos. En cuanto a recursos educativos que apoyan la internacionalización en el aula, el cuerpo docente usa blogs, bases de datos, software educativo, videos y artículos especializados. Se hace necesario establecer objetivos de internacionalización claros y estrategias de seguimiento para poder medir los avances en los procesos de internacionalización en el aula.

Palabras clave: internacionalización curricular, educación superior, formación docente, calidad, acreditación.

\begin{abstract}
Internationalization is a process that supports the development of the quality of institutions. In Early Childhood Education programs, which train the teachers of the next generations, internationalization plays an increasingly important role as globalization and the knowledge society have pressure teaching. Following this idea, this article presents the results of one of the objectives of a doctoral thesis, concentrating on understanding how internationalization is carried out in the classroom in Bachelor programs in Early Childhood Education in the institutions Corporación Universitaria del Caribe and Universidad de La Guajira in Colombia. This analysis was carried out taking into account the guidelines for assuring the quality of programs in Colombia and the Guide for Quality in Internationalization proposed by the European Council of Accreditation Agencies. Through 17 interviews with teachers, the data was collected. It is observed that in terms of teaching methodologies, active pedagogies, collaborative online learning with international peers, as well as debates and problem-based learning are prioritized. Projects, workshops, presentations and written tests are used to evaluate. As for educational resources that support internationalization in the classroom, teachers use blogs, databases, educational software, videos, and specialized articles. It is necessary to establish clear internationalization objectives and follow-up strategies in order to measure progress in the internationalization processes in the classroom.
\end{abstract}

Keywords: curriculum internationalization, higher education, teacher training, quality, accreditation.

La internacionalización en el aula en Licenciaturas en Educación Infantil en el Caribe colambiana: una perspectiva dacente Luisa F. Echeverría King, Fuensanta Hernández-Pinal, Diana Laga de Vergara

DDI: http://dx.dai.org/l0.22458/caes.vlliz.2831

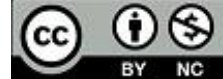

Artícula prategida par licencia Sreative Cammans 


\section{Introducción}

La internacionalización es actualmente un aspecto central en los programas de educación superior y lineamiento relevante ante agencias acreditadoras. Es una respuesta natural de la educación superior a la globalización de los mercados y una necesidad ante la sociedad del conocimiento, la cual busca conectar a los países y sus sistemas educativos al compartir proyectos, programas y colaboraciones conjuntas, incluso De Zan et al. (2011) exponen que la universidad, al ser una entidad que se conformó y masificó gracias a la globalización, tiene el deber de jugar un rol proactivo frente a los procesos de internacionalización de la educación. La internacionalización es descrita por Echeverría y Lafont (2018) como un proceso transversal a las funciones misionales de las instituciones, el cual busca responder a dinámicas como la globalización y cuya finalidad puede ser el mejoramiento de la calidad en la ejecución de las funciones misionales en las instituciones. Por muchos años, la internacionalización se ha visto en las instituciones de educación superior (IES) como una serie de actividades generadas por docentes u oficinas de internacionalización sin responder a planes concretos, sin embargo el experto De Wit (2011) explica que la internacionalización ha pasado de ser un proceso reactivo, a estar orientado de forma proactiva en las instituciones y encontrarse generalizado; este proceso, según el autor, depende del contexto y de los enfoques propios institucionales de qué y cómo internacionalizar.

Como respuesta a la internacionalización de la educación se observa la internacionalización de los currículos, en la cual el profesorado juega un rol importante, especialmente desde el aspecto microcurricular. Probablemente la definición más conocida es la de la autora australiana Leask (2009), quien expone que la internacionalización curricular busca introducir dimensiones interculturales, internacionales o globales en el contenido curricular, así como en los objetivos de aprendizaje, acciones de evaluación, metodologías docente y los servicios de apoyo que ofrece una institución determinada. En todo caso las dinámicas de la

La internacionalización en el aula en Licenciaturas en Educación Infantil en el Caribe colombiana: una perspectiva dacente Luisa F. Echeverría King, Fuensanta Hernández-Pinal, Diana Lago de Vergara

DDI: http://dx.dai.org/I0.22458/caes.vlli2.2831

(c)

Artícula prategida por licencia Ereative Cammans 
internacionalización entran en tensión con las disciplinas, por lo cual se insta a pensar en estrategias de internacionalización para programas académicos de estudio puntuales.

Este artículo busca presentar los resultados preliminares de una tesis doctoral sobre la internacionalización curricular en programas de Licenciatura en Educación Infantil con base en entrevistas efectuadas a docentes de estos programas en las instituciones Universidad de la Guajira y Corporación Universitaria del Caribe (Cecar), ambas ubicadas en el Caribe colombiano; se tienen en cuenta los referentes para el aseguramiento de la calidad en Colombia y los lineamientos para la calidad de la internacionalización del Consejo Europeo de Agencias de Acreditación (ECA). Este artículo se enfocará en presentar según la información levantada en las entrevistas, cómo se desarrolla la internacionalización en el aula y en el proceso enseñanza-aprendizaje en las licenciaturas participantes en el estudio, desde una perspectiva docente; se consideran los mencionados referentes de calidad como énfasis para el análisis de la información.

El artículo se divide de la siguiente manera: primeramente, se presentarán algunos referentes teóricos sobre la internacionalización curricular. Después, se expondrán las directrices para el aseguramiento de la calidad de programas académicos en Colombia y su relación con la internacionalización, así como los lineamientos para la calidad en la internacionalización del Consejo Europeo para la Acreditación. Luego se expone información sobre los programas objeto de estudio, así como lineamientos curriculares de las instituciones Cecar y la Universidad de la Guajira, que sostienen la internacionalización de los microcurrículos. Seguidamente, se expone la metodología de investigación y los resultados evidenciados, de cómo se efectúa la internacionalización en el aula y en el proceso enseñanza-aprendizaje en los programas de Licenciatura en Educación Infantil en las mencionadas IES. Por último, se describen algunas conclusiones y recomendaciones que pueden apoyar al profesorado de

La internacionalización en el aula en Licenciaturas en Educación Infantil en el Caribe colombiana: una perspectiva dacente Luisa F. Echeverría King, Fuensanta Hernández-Pinal, Diana Lago de Vergara

DDI: http://dx.dai.org/I0.22458/caes.vlli2.2831

(c) (i) (8)

Artícula protegida par licencia Creative Cammans 
programas similares en otras IES a implementar acciones de internacionalización a nivel microcurricular.

\section{Referentes teóricos: la internacionalización curricular}

El currículo internacional es de hecho el más importante aspecto de los sistemas de educación superior internacionalizados (Ardakani et al., 2011). Crosling et al. (2008) resaltan que la internacionalización curricular es una estrategia de las universidades, la cual tiene el objetivo de preparar a sus futuros egresados para laborar en entornos cada vez más globalizados. Hoff y Kappler (2005) explican la importancia del aprendizaje intercultural, especialmente de cara a la preparación de experiencias de movilidad internacional. Introducir a los estudiantes a los conceptos y experiencias asociadas al aprendizaje intercultural, prepara a los estudiantes según los autores, para trabajar, vivir y estudiar en entornos internacionales o diversos.

Leask (2014) explica que los enfoques en cada una de las disciplinas implementadas en la internacionalización curricular pueden ser diversos, sin embargo, lo más relevante es el aprendizaje del alumnado. Esto ubica en el centro de la internacionalización del currículo al profesorado y sus áreas de enseñanza. Esta idea es reforzada por Peña y Aponte (2018), quienes explican que el conjunto docente tiene el reto de incluir las dimensiones internacionales, interculturales e interdisciplinarias a los cursos, sus entornos y métodos de enseñanza. Según Gamboa et al. (2016), el profesorado es el encargado de formar al estudiantado de forma integral, con el fin de que el futuro egresado pueda ejercer la ciudadanía de forma consciente. Sin embargo, la responsabilidad de la internacionalización no solo recae en el personal docente. Peña y Aponte (2018) ejecutan un especial énfasis en la idea de que la internacionalización se implementa gracias a una articulación de los diferentes actores de la internacionalización, donde resaltan especialmente la figura del

La internacionalización en el aula en Licenciaturas en Educación Infantil en el Caribe colombiana: una perspectiva dacente Luisa F. Echeverría King, Fuensanta Hernández-Pinal, Diana Lago de Vergara

DDI: http://dx.dai.org//0.22458/caes.vlli2.2831

(c)

Artícula prategida par licencia Creative Cammans 
personal de la administración y directivos como apoyo central en la implementación de este proceso.

Según Sawir (2013), los académicos de diversas disciplinas pueden tener concepciones diferentes frente a la internacionalización curricular, sin embargo, un punto en común que tienen todos es el ejercicio pedagógico, el cual juega un rol central en este proceso. Por su parte, Arango y Acuña (2018) explican la importancia de la flexibilidad curricular de cara a la internacionalización, sin olvidar la pertinencia de los currículos e incluyendo medios virtuales para la interacción docente-estudiante. Estos autores explican igualmente que las instituciones enfocadas en implementar la internacionalización curricular deberán establecer planes y acciones medibles con indicadores de resultado e impacto, desarrollando asimismo seguimiento correspondiente a las metas trazadas. Peña (2019) efectúa un especial énfasis en la importancia del uso intencional de plataformas digitales para la instrucción y transmisión de información, entre autores internos y externos de la internacionalización. La autora comenta que "la intencionalidad consiste en crear nuevas redes de conexiones y aprendizaje a fin de construir visiones alternativas para la internacionalización del currículo en la educación superior" (p. 4).

\section{Referentes para la calidad}

Requerimientos para el aseguramiento de la calidad de la internacionalización en los programas académicos: Lineamientos de Colombia

En el 2019 se han actualizado los requerimientos para la obtención del registro calificado (condiciones mínimas de funcionamiento) en los programas de educación superior. Con base en lo expresado en el Decreto 1330 de 2019, en los aspectos curriculares, se evidencia que los programas académicos en Colombia deben articular elementos como la flexibilidad curricular y el perfil de egreso de acuerdo con habilidades requeridas por el contexto nacional e internacional. Igualmente, este decreto insta a los programas a trabajar

La internacionalización en el aula en Licenciaturas en Educación Infantil en el Caribe colombiana: una perspectiva dacente Luisa F. Echeverría King, Fuensanta Hernández-Pinal, Diana Lago de Vergara

DDI: http://dx.dai.org//0.22458/caes.vlli2.2831

(c)

Artícula prategida par licencia Creative Cammans 
por un currículo internacionalizado que fomente la inserción de profesores y estudiantes en ambientes internacionales y el aprendizaje de una segunda lengua. Del mismo modo, los programas deberán establecer mecanismos que fomenten el relacionamiento con el sector externo, con el fin de ampliar su función e impacto social. Asimismo, la investigación generada de un programa determinado deberá dar respuesta a situaciones y retos de la región, nación o problemas globales. Por último, este decreto enfatiza que los programas deben contar con medios educativos e infraestructura que dinamicen y soporten el uso de nuevas tecnologías que fomenten el desarrollo de las funciones misionales del programa.

Tabla 1. Condiciones institucionales y de programa para obtener registro calificado en Colombia

\begin{tabular}{|c|c|}
\hline $\begin{array}{c}\text { Condiciones institucionales } \\
\end{array}$ & Condiciones programa académico \\
\hline $\begin{array}{l}\text { 1. Mecanismos de selección y evaluación de } \\
\text { estudiantes y profesores } \\
\text { a) Mecanismos de selección y evaluación de } \\
\text { estudiantes } \\
\text { b) Mecanismos de selección y evaluación de profesores }\end{array}$ & 1. Denominación del programa \\
\hline $\begin{array}{l}\text { 2. Estructura administrativa y académica } \\
\text { a) Gobierno Institucional y rendición de cuentas. } \\
\text { b) Políticas Institucionales } \\
\text { c) Gestión de la Información } \\
\text { d) Arquitectura Institucional }\end{array}$ & 2. Justificación del Programa \\
\hline 3. Cultura de autoevaluación & $\begin{array}{l}\text { 3. Aspectos Curriculares } \\
\text { a) Componentes formativos } \\
\text { b) Componentes pedagógicos } \\
\text { c) Componentes de interacción } \\
\text { d) Conceptualización teórica y epistemológica del } \\
\text { programa } \\
\text { e) Mecanismos de evaluación }\end{array}$ \\
\hline 4. Programa de Egresados & $\begin{array}{l}\text { 4. Organización de las actividades académicas y } \\
\text { proceso formativos. }\end{array}$ \\
\hline 5. Modelo de Bienestar & $\begin{array}{l}\text { 5. Investigación, innovación y/o creación artísticas } \\
\text { y cultural }\end{array}$ \\
\hline \multirow{4}{*}{$\begin{array}{l}\text { 6. Recursos suficientes para garantizar el cumplimiento } \\
\text { de las metas } \\
\text { a) Gestión del Talento Humano } \\
\text { b) Recursos físicos y Tecnológicos } \\
\text { c) Recursos financieros }\end{array}$} & 6. Relación con el sector externo \\
\hline & 7. Profesores \\
\hline & 8. Medios Educativos \\
\hline & 9. Infraestructura física y tecnológica \\
\hline
\end{tabular}

Fuente: (Ministerio de Educación Nacional, 2019)

La internacionalización en el aula en Licenciaturas en Educación Infantil en el Caribe colombiana: una perspectiva dacente Luisa F. Echeverría King, Fuensanta Hernández-Pinal, Diana Laga de Vergara

DDI: http://dx.dai.org/I0.22458/caes.vlli2.2831

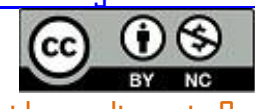

Artícula prategida por licencia Ereative Cammans 
Lineamientos para la calidad en la internacionalización promovidos por el Consorcio Europeo de Agencias de Acreditación

Al revisar referentes internacionales de relevancia para el presente trabajo encontramos que en el 2015 la Asociación Europea para el Aseguramiento de la Calidad, emitió un documento denominado "Criterios y directrices para el Aseguramiento de la Calidad en el Espacio Europeo de Educación Superior". Este documento, cuyo interés principal es brindar orientaciones para el aseguramiento de la calidad en todos los países que hacen parte del espacio europeo del conocimiento y "contribuir al entendimiento común del aseguramiento de la calidad de la enseñanza y del aprendizaje en cualquier país y entre todas las partes interesadas" (Consorcio Europeo de Agencias de Acreditación, 2015, p. 7) ha sido la base para que el Consorcio Europeo de Agencias de Acreditación (ECA) creara en el 2015 los Lineamientos para la Medición de la Calidad en la Internacionalización, Frameworks for the Assessment of Quality in Internationalisation y su respectiva Guía para la Calidad en la Internacionalización en el 2017, Guide to Quality in Internationalization. Estos lineamientos aplican para la revisión de estándares de calidad en la internacionalización a nivel de programa o institucional. Según lo expuesto en los pilares de esta guía de la calidad de la internacionalización, encontramos los siguientes criterios de evaluación para programas académicos:

La internacionalización en el aula en Licenciaturas en Educación Infantil en el Caribe colombiana: una perspectiva dacente Luisa F. Echeverría King, Fuensanta Hernández-Pinal, Diana Laga de Vergara

DOI: http://dx.dai.org/l0.22458/caes.vlliz.2831

(c) () (5)

Artícula protegida par licencia Creative Cammans 
Tabla 2. Lineamientos para la calidad en la internacionalización de programas académicos según el Consorcio Europeo de Agencias de Acreditación (2017)

\begin{tabular}{|c|c|}
\hline Lineamientos & Descripción \\
\hline $\begin{array}{l}\text { Internacionalización intencionada } \\
\text { Intended Internationalization }\end{array}$ & $\begin{array}{l}\text { Este eje revisa cómo están planteadas las metas de } \\
\text { internacionalización en un programa académico determinado; } \\
\text { igualmente analiza cuáles son las estrategias para monitorear } \\
\text { el alcance de las metas propuestas y el impacto de la } \\
\text { internacionalización en la calidad de la educación. }\end{array}$ \\
\hline $\begin{array}{l}\text { Aprendizaje internacional e intercultural } \\
\text { International and Intercultural learning }\end{array}$ & $\begin{array}{l}\text { Para este eje es central la revisión de cómo los resultados de } \\
\text { aprendizaje interculturales e internacionales están } \\
\text { relacionados con los objetivos de internacionalización } \\
\text { globales del programa, cómo funciona la evaluación de los } \\
\text { resultados de aprendizaje interculturales e internacionales y el } \\
\text { respectivo alcance de estos al culminar el programa. }\end{array}$ \\
\hline $\begin{array}{l}\text { Enseñanza y aprendizaje } \\
\text { Teaching and learning }\end{array}$ & $\begin{array}{l}\text { La internacionalización debe ser transversal al proceso } \\
\text { enseñanza-aprendizaje, por esto en el marco de este eje se } \\
\text { revisan principalmente tres elementos: el currículo, el cual } \\
\text { deberá estar alineado con los objetivos de } \\
\text { internacionalización del programa y los resultados de } \\
\text { aprendizaje internacionales e interculturales. Se evalúa } \\
\text { igualmente las metodologías de enseñanza-aprendizaje y los } \\
\text { ambientes de aprendizaje, los cuales deben coadyuvar al } \\
\text { alcance de los resultados de aprendizaje internacionales e } \\
\text { interculturales. }\end{array}$ \\
\hline $\begin{array}{l}\text { Personal académico y administrativo } \\
\text { Staff }\end{array}$ & $\begin{array}{l}\text { Otro de los elementos centrales evaluados es el personal } \\
\text { académico y administrativo, el cual está a cargo del programa. } \\
\text { Este personal debe contar en el mejor de los casos con } \\
\text { experiencia internacional, debe dominar idiomas extranjeros, } \\
\text { contar con competencias interculturales y fungir como } \\
\text { plataforma para que la calidad del proceso enseñanza- } \\
\text { aprendizaje, así como los demás servicios que ofrezca un } \\
\text { programa determinado, puedan estar al alcance de todo el } \\
\text { estudiantado. }\end{array}$ \\
\hline $\begin{array}{l}\text { Estudiantes } \\
\text { Students }\end{array}$ & $\begin{array}{l}\text { El grupo de estudiantes adscritos al programa debe tener no } \\
\text { solo la oportunidad de beneficiarse con aprendizaje } \\
\text { intercultural e internacional, sino que también deberá poder } \\
\text { acceder a servicios como movilidad académica estudiantil y } \\
\text { otros servicios que internacionalicen su experiencia de estudio } \\
\text { en el programa. }\end{array}$ \\
\hline
\end{tabular}

Fuente: Consorcio Europeo de Agencias de Acreditación (2017)

La internacionalización en el aula en Licenciaturas en Educación Infantil en el Caribe colombiana: una perspectiva dacente Luisa F. Echeverría King, Fuensanta Hernández-Pinal, Diana Lago de Vergara

DDI: http://dx.dai.org//0.22458/caes.vlli2.2831

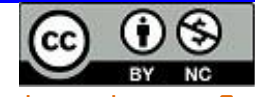

Artícula protegida par licencia Creative Cammans 
Como se puede observar, los lineamientos planteados en la mencionada Guía para la Calidad de la Internacionalización, tienen como objetivo principal analizar las metas y objetivos planteados de internacionalización de programas, así como los resultados de aprendizaje internacionales e interculturales, las formas de enseñanza para alcanzar estos resultados de aprendizaje, currículo, la formación del personal docente y administrativo, su experiencia internacional y los servicios ofertados para estudiantes, que complementan su experiencia internacional dentro o fuera del campus. Para el presente artículo es de especial relevancia los aspectos asociados a la categoría de "enseñanza y aprendizaje".

\section{Contextos institucionales}

A continuación, se describen los contextos institucionales y directrices curriculares frente a la internacionalización, los cuales se observan en las instituciones y programas objeto de estudio.

Corporación Universitaria del Caribe (Cecar) y su programa denominado Licenciatura en Pedagogía Infantil

La Corporación Universitaria del Caribe (Cecar) se trata de una institución localizada en el departamento de Sucre (Colombia), en la ciudad de Sincelejo. Ofrece programas presenciales, a distancia y virtuales; también tiene presencia en las ciudades colombianas de Montería y Villavicencio. La institución universitaria cuenta con más de 30 años de haberse fundado y a través de los años ha trabajado para convertirse en una entidad de importancia en la región y con gran proyección social. Con base en sus proyecciones institucionales, Cecar busca formar a una ciudadanía crítica, creativa e innovadora; para ello, tiene tres ejes de trabajo: la calidad educativa, la investigación y la internacionalización (Cecar, 2020).

La internacionalización en el aula en Licenciaturas en Educación Infantil en el Caribe colombiana: una perspectiva dacente Luisa F. Echeverría King, Fuensanta Hernández-Pinal, Diana Laga de Vergara

DDI: http://dx.dai.org/I0.22458/caes.vlli2.2831

(c) () (5)

Artícula prategida par licencia Creative Cammans 
Desde su Proyecto Educativo Institucional, Cecar promueve que todo diseño curricular tendrá en cuenta las tendencias de las disciplinas y profesiones a nivel internacional, por lo cual los estudios comparativos son relevantes para esta institución como una forma de impulsar el intercambio y las relaciones con otros países del mundo:

El diseño y la planeación curricular de los programas académicos ofertados por Cecar, en todos los niveles y modalidades, tienen en cuenta las tendencias de formación y titulación en las disciplinas y profesiones en contextos internacionales; fomentándose los estudios comparativos entre programas de la misma naturaleza ofertados en otros países o regiones del mundo, para propiciar el intercambio y la movilidad de estudiantes y docentes. (Cecar, 2017, p. 29)

Cecar apertura el programa de Licenciatura en Pedagogía Infantil, en enero de 2016, para apoyar de esta manera a la formación de recurso humano que pueda fungir como docente, investigador o directivo de instituciones de atención a la primera infancia, en especial en la región Caribe de Colombia. En la actualidad, el programa cuenta con 122 estudiantes en la modalidad presencial (Cecar, 2019).

El programa cuenta con los siguientes objetivos:

- Generar espacios académicos para la construcción, aplicación y sistematización de proyectos pedagógicos innovadores en el área de educación infantil.

- Desarrollar competencias investigativas en los educadores infantiles que les permitan generar proyectos pedagógicos enfocados a la primera infancia, a las políticas educativas y a la innovación en educación infantil.

- Formar licenciados en educación infantil con pensamiento crítico, comprometidos con el desarrollo integral, protección y garantía de los derechos de infancia. (Cecar, 2019, p. 8)

La internacionalización en el aula en Licenciaturas en Educación Infantil en el Caribe colombiana: una perspectiva dacente Luisa F. Echeverría King, Fuensanta Hernández-Pinal, Diana Laga de Vergara

DDI: http://dx.dai.org/I0.22458/caes.vlli2.2831

(c) (i) (\$)

Artícula prategida par licencia Lreative Cammans 
Según el Proyecto Educativo del Programa de la Licenciatura en Pedagogía Infantil de Cecar (2019), la formación en el aula se deberá desarrollar con un enfoque participativo, donde se articule la teoría con la práctica y favoreciendo el aprendizaje autónomo del estudiante:

En concordancia con los propósitos educativos y las competencias que se busca desarrollar en los y las estudiantes, el proceso académico-pedagógico y didáctico se constituye en un espacio participativo para la Motivación, Conceptualización, Construcción y aplicación y Evaluación de conocimientos en las asignaturas teóricas, teórico-prácticas y prácticas. De este modo, las actividades académico pedagógicas que posibilitan el desarrollo de las competencias de los/as estudiantes en el saber, saber hacer y saber Ser, se organizan como trabajo directo, asistido e independiente, centrando el interés en el aprendizaje autónomo, conforme a lo establecido en el Modelo pedagógico Institucional. (p. 27)

En este sentido se observa que Cecar promueve enfoques y tendencias internacionales para la planeación curricular, así como fomenta desde el Programa de Pedagogía Infantil innovaciones desde el aula, las cuales le apuntarán al desarrollo de competencias y el fomento de la autonomía en el aprendizaje.

\section{Universidad de la Guajira y su programa Licenciatura en Educación Infantil}

La Universidad de la Guajira se crea por medio del Decreto 523 de 1976, adscribiéndola a la Secretaría de Educación Departamental de la Guajira. Su sede principal queda en la ciudad de Riohacha, la cual es la capital del departamento de la Guajira (Colombia). Cabe resaltar que este departamento se encuentra en la frontera con Venezuela. Al revisar los objetivos plasmados en el Decreto 523 de 1976 para la Universidad de la Guajira, se observa que esta entidad fomentará el desarrollo económico y cultural de la región por medio de sus funciones sustantivas (formación, investigación y extensión). Asimismo se expresa en este decreto la

La internacionalización en el aula en Licenciaturas en Educación Infantil en el Caribe colombiana: una perspectiva dacente Luisa F. Echeverría King, Fuensanta Hernández-Pinal, Diana Laga de Vergara

DDI: http://dx.dai.org/I0.22458/caes.vlli2.2831

(c)

Artícula prategida par licencia Creative Cammans 
importancia de incluir en las acciones formativas a la población indígena localizada en el departamento.

En su Proyecto Educativo Institucional la Universidad de la Guajira establece que, al ser una universidad ubicada en zona de frontera entre Colombia y Venezuela, y en una región con importante presencia de la etnia Wayuu, su proyecto educativo cuenta con un enfoque multiétnico y multicultural, esto favorece no solo el respeto hacia las culturas ancestrales sino también un trabajo desde la formación que le aporte a la integración con el vecino país de Venezuela (Universidad de la Guajira, 2017):

La Universidad de La Guajira se plantea en este proyecto educativo desde lo nacional y lo internacional como una síntesis cultural de la región legada por el pueblo wayuu, por consiguiente en su conocimiento podrían residir las respuestas base de la acción para construir una región que satisfaga las aspiraciones sociales e individuales del hombre de frontera, es decir el hombre internacional, contribuyendo a fortalecer la unidad nacional con esquemas autónomos y eficaces de verdadera integración binacional e internacional. (p. 8)

El Programa de Educación Infantil se crea en el 2001 como respuesta a una reorganización de la Facultad de Educación, en la cual se evidenció la necesidad de formar a docentes que pudiesen atender a la población infantil en la región. En el marco de procesos recientes de autoevaluación adopta la nueva denominación sugerida por el Ministerio de Educación Nacional: Licenciatura en Educación Infantil. (Universidad de la Guajira, 2016). Al momento de la recogida de datos, la sede Riohacha contaba con 454 estudiantes.

Al valorar esta información, el programa de Licenciatura en Educación Infantil se propone formar (Universidad de la Guajira, 2016):

La internacionalización en el aula en Licenciaturas en Educación Infantil en el Caribe colombiana: una perspectiva dacente Luisa F. Echeverría King, Fuensanta Hernández-Pinal, Diana Lago de Vergara

DDI: http://dx.dai.org/I0.22458/caes.vlli2.2831

(c) 
-Licenciados y licenciadas con un alto nivel de competencia en el campo de la pedagogía y la didáctica comprometidos con el cambio social y el bienestar integral de la primera infancia.

-Profesionales éticos con pensamiento crítico y reflexivo; personas sensibles, creativas, equilibradas y asertivas; para generar procesos de interacción con sus pares, estudiantes, sus familias y sus entornos; reconociendo las potencialidades del "otro" con el fin de aprender a "hacer" y aprender a "convivir" dentro de un contexto multicultural.

-Investigadores y gestores sociales que retroalimenten constantemente su práctica pedagógica, que participen en las redes académicas nacionales e internacionales, que aporten nuevos conocimientos desde la interdisciplinariedad y que cualifiquen el diseño e implementación de modelos pedagógicos innovadores. (p. 25)

Según el Proyecto Educativo del Programa el currículo se genera y se re-construye con base en las necesidades de la disciplina y contexto, teniendo en cuenta especialmente una pedagogía con enfoque intercultural dadas la condiciones del entorno. Asimismo se fomenta la interacción permanente entre el docente y sus estudiantes por medio de las metodologías docente:

En este orden de ideas, el currículo se construye y re-construye desde las disciplinas necesarias para el estudio y comprensión de la educación infantil, pero también articula las diversas concepciones de infancia y problemáticas que puedan estar presentes en el contexto de desarrollo de este programa de formación, mediante una pedagogía para la interculturalidad que permea los cuatro campos de formación. Esto permite desarrollar en el estudiante competencias a través de ciclos estratégicos en los que se potencian y dosifican los contenidos y que se

La internacionalización en el aula en Licenciaturas en Educación Infantil en el Caribe colombiana: una perspectiva dacente Luisa F. Echeverría King, Fuensanta Hernández-Pinal, Diana Laga de Vergara

DDI: http://dx.dai.org/I0.22458/caes.vlli2.2831

(c) 
dinamizan a través de estrategias metodológicas mediadas por la permanente interacción entre docentes y estudiantes. (Universidad de la Guajira, 2016, p. 79)

Con base en estos contextos institucionales y programáticos se revisa a continuación la metodología de investigación utilizada para comprender cómo se desarrolla la internacionalización curricular en los programas de Educación Infantil objeto de estudio.

\section{Metodología}

\section{Objetivo}

Este artículo presenta los resultados de uno de los objetivos de una tesis doctoral, el cual le apunta a comprender cómo se desenvuelve la internacionalización en el aula y en el proceso enseñanza-aprendizaje en Licenciaturas en Educación Infantil, de las instituciones Universidad de la Guajira y Corporación Universitaria del Caribe en Colombia, así como recoger sugerencias por parte del cuerpo docente, para mejorar la internacionalización del proceso enseñanza-aprendizaje en estos programas de estudio.

\section{Diseño}

Con el fin de indagar el objetivo presentado, se desarrolla un estudio de corte cualitativo fenomenológico, se toma en cuenta como referente central a docentes de programas de Licenciatura en Educación Infantil, de instituciones objeto de estudio; este diseño se escogió dado que por medio de la fenomenología, se consideran las experiencias de las personas entrevistadas. Según Colás et al. (2009), "el método fenomenológico se centra en las descripciones que los sujetos hacen de sus vivencias" (p. 102), por lo que se consigna información propia de la experiencia docente de los entrevistados. Hernández-Pina y Maquilón Sánchez (2015) expresan igualmente, que la investigación cualitativa "se define como aquella en la que los investigadores recopilan los datos en situaciones reales mediante

La internacionalización en el aula en Licenciaturas en Educación Infantil en el Caribe colombiana: una perspectiva dacente Luisa F. Echeverría King, Fuensanta Hernández-Pinal, Diana Laga de Vergara

DDI: http://dx.dai.org//0.22458/caes.vlli2.2831

$$
\text { (c) }(\text { i) }(2)
$$


la interacción con las personas que participarán, siendo fuente de los datos necesarios para llevar a cabo la investigación" (p. 28).

Instrumento de recogida de información

Se efectuaron entrevistas semiestructuradas con preguntas abiertas, para un mejor flujo de la conversación. Creswell (2012) expone que las preguntas abiertas en las entrevistas permiten que las personas participantes puedan expresar sus experiencias y vivencias sin restricciones.

Tabla 3. Dimensiones guía de entrevista para docentes

\begin{tabular}{|l|l|}
\hline \multicolumn{1}{|c|}{ Dimensiones } & \multicolumn{1}{|c|}{ Descripción de la dimensión } \\
\hline $\begin{array}{l}\text { 1. Conceptualización sobre la } \\
\text { internacionalización de la educación superior }\end{array}$ & $\begin{array}{l}\text { Se indaga sobre la conceptualización de la } \\
\text { internacionalización para los docentes, teniendo en cuenta } \\
\text { sus experiencias personales y profesionales }\end{array}$ \\
\hline $\begin{array}{l}\text { 2. Internacionalización curricular: acciones } \\
\text { lideradas por docentes }\end{array}$ & $\begin{array}{l}\text { Se indaga información, sobre cómo se lleva a cabo la } \\
\text { internacionalización en el aula en las Licenciaturas en } \\
\text { Educación Infantil, revisando aspectos como el proceso } \\
\text { enseñanza-aprendizaje, los métodos de evaluación y los } \\
\text { medios educativos, con base en los lineamientos de } \\
\text { aseguramiento de la calidad en Colombia y el referente de la } \\
\text { Guía para la Calidad de la Internacionalización del ECA. }\end{array}$ \\
\hline $\begin{array}{l}\text { 3. Sugerencias para el mejoramiento de la la } \\
\text { internacionalización del proceso enseñanza- } \\
\text { aprendizaje }\end{array}$ & $\begin{array}{l}\text { Se requiere información sobre cómo el entrevistado propone } \\
\text { puede mejorar la internacionalización desde el } \\
\text { microcurrículo. }\end{array}$ \\
\hline
\end{tabular}

Fuente: Elaboración propia

Análisis de la información

Para el análisis de las entrevistas, se ha priorizado el análisis cualitativo, formando categorías y subcategorías para organizar la información obtenida de las entrevistas; para esto se utilizó el apoyo del software MAXQDA. Mayring (2002), expone que el análisis cualitativo es una

La internacionalización en el aula en Licenciaturas en Educación Infantil en el Caribe colambiana: una perspectiva dacente

Luisa F. Echeverría King, Fuensanta Hernández-Pinal, Diana Laga de Vergara

DDI: http://dx.dai.org//0.22458/caes.vlli2.2831

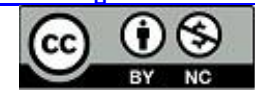

Artícula prategida par licencia Creative Cammans 
metodología la cual busca analizar los textos de forma sistemática, compilando información relevante en categorías formadas con ayuda de la teoría y demás documentos de interés para la investigación.

\section{Participantes}

Se entrevistaron en total 17 docentes de dos instituciones objeto de estudio, 10 en la Universidad de la Guajira y 7 de Cecar, quienes ejercen docencia en el marco de programas de Licenciatura en Educación Infantil. Las entrevistas se llevaron a cabo durante mayo de 2019. Después de su elaboración, se procedió a transcribir el material y organizarlo por categorías y subcategorías, para la organización de los datos recogidos.

A continuación se presentan los resultados correspondientes.

\section{Presentación de resultados y discusión}

Al indagar sobre la definición de internacionalización para los docentes de la Licenciatura en Pedagogía Infantil de Cecar, se observa según la figura 1, que los profesores relacionan este concepto con adaptaciones curriculares internacionales, intercambios culturales $\mathrm{y}$ de experiencias entre pares, así como la creación de conexiones entre los modelos y estrategias pedagógicas internacionales y las propias del contexto. Asimismo, se observa que indican que la internacionalización fomenta el aprendizaje entre pares, amplía los horizontes en la educación superior y en el área disciplinar en particular, así como impulsa la generación de alianzas con IES internacionales, lo cual se relaciona con el concepto de internacionalización promovido por Echeverría y Lafont (2018) al ser un proceso transversal y dinámico.

La internacionalización en el aula en Licenciaturas en Educación Infantil en el Caribe colombiana: una perspectiva dacente Luisa F. Echeverría King, Fuensanta Hernández-Pinal, Diana Laga de Vergara

DDI: http://dx.dai.org//0.22458/caes.vlli2.2831

(c) $\underset{\mathrm{BY}}{\mathrm{BY}}(\mathrm{sc}$ 
Figura 1. Definición de internacionalización para docentes de Cecar

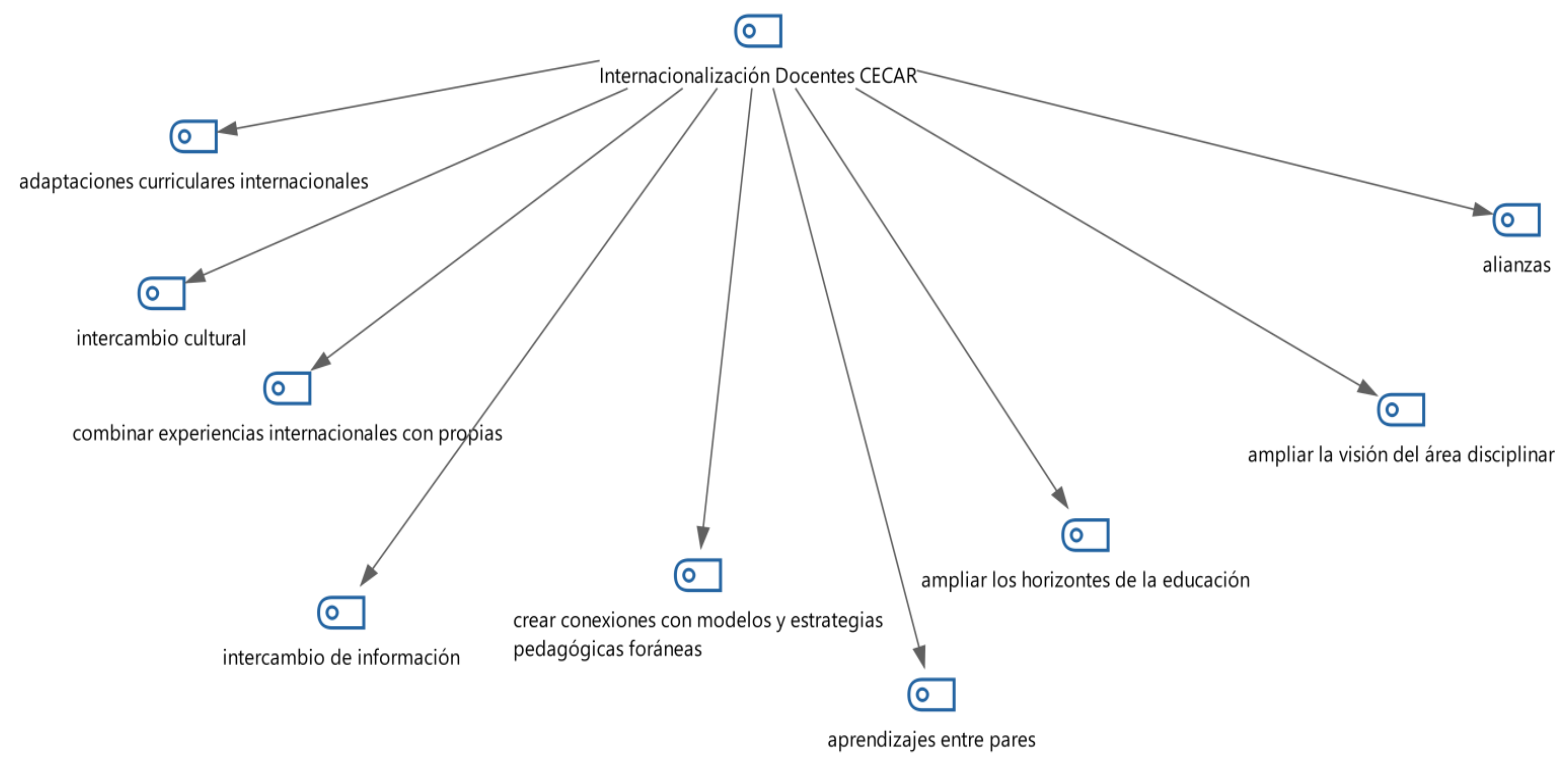

El profesorado de la Uniguajira relaciona la internacionalización con referentes foráneos según las diversas temáticas de las disciplinas, comparaciones curriculares, culturas y lenguas extranjeras, así como con aspectos asociados a la visibilidad internacional del programa o de la IES. Cabe resaltar que la interculturalidad es un aspecto muy importante en la Uniguajira por su situación geográfica, en este sentido el aprendizaje intercultural juega un rol importante especialmente de cara a la preparación frente a intercambios o movilidades internacionales (Hoff y Kappler, 2005). Introducir al estudiantado a los conceptos y experiencias asociadas con el aprendizaje intercultural, lo prepara, según los autores, para trabajar, vivir y estudiar en entornos internacionales o diversos. También se observa la relación con capacitaciones internacionales, estímulos para reconocer el trabajo del cuerpo docente a nivel internacional, así como espacios de intercambio con pares internacionales

La internacionalización en el aula en Licenciaturas en Educación Infantil en el Caribe colambiana: una perspectiva dacente Luisa F. Echeverría King, Fuensanta Hernández-Pinal, Diana Lagu de Vergara

DDI: http://dx.dai.org/I0.22458/caes.vlli2.2831

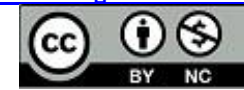

Artícula protegida par licencia Creative Cammans 
que apoyen el aprendizaje (ver figura 2). En este sentido estas definiciones van de la mano con lo planteado en el Proyecto Educativo Institucional de la Uniguajira (2017), el cual le apunta a la formación de un ser humano de frontera, internacional, que reconozca su contexto y pueda darle soluciones a su entorno desde la integración de lo foráneo.

Figura 2. Definición de internacionalización para docentes de la Uniguajira

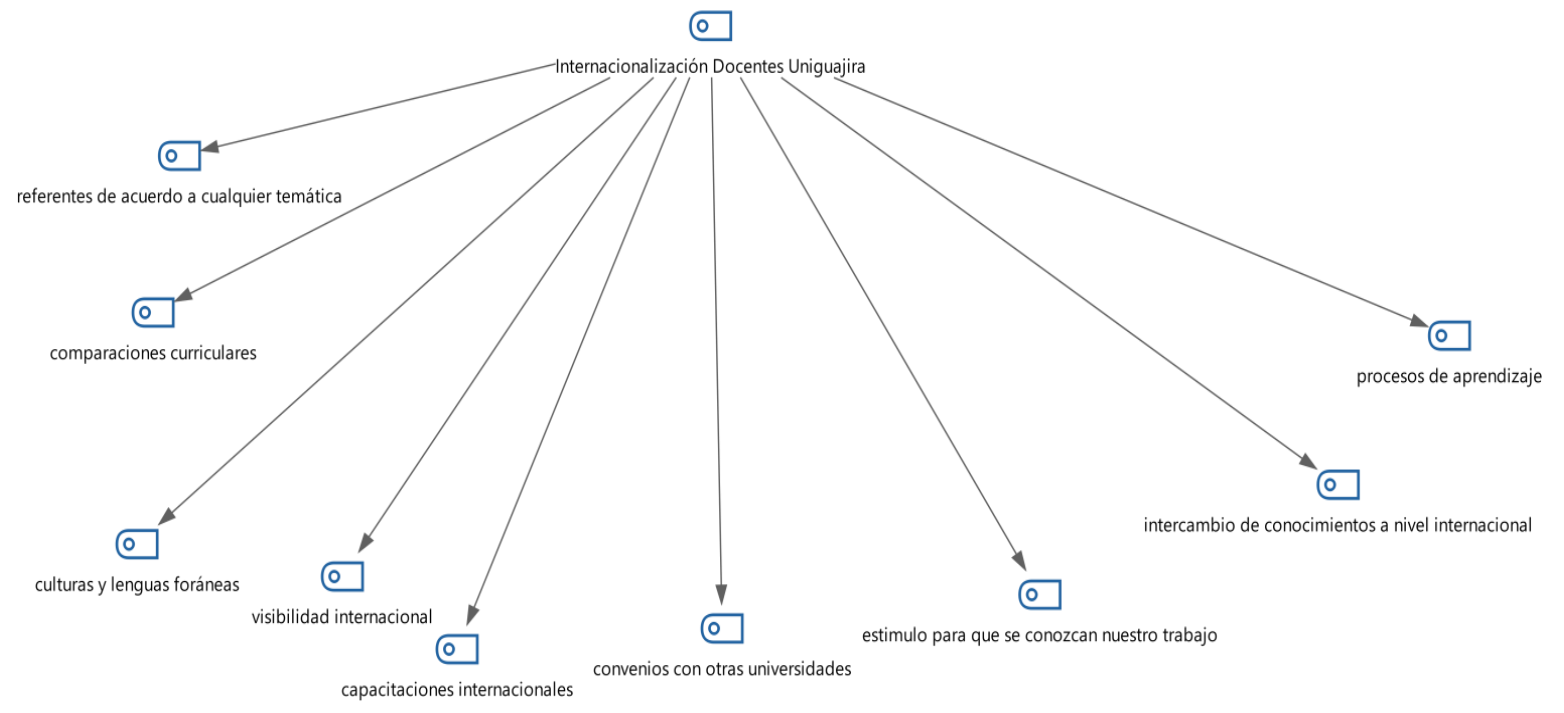

Al revisar la internacionalización del proceso enseñanza-aprendizaje en Cecar, en el marco de la Licenciatura en Pedagogía Infantil se observan diversas metodologías docentes, como los debates, las clases magistrales, clases espejo ${ }^{4}$, así como pedagogías activas como lo son estrategias pedagógicas como el aprendizaje basado en proyectos y problemas. Asimismo el

\footnotetext{
${ }^{4}$ Las clases espejo son formatos de intercambio virtual en el marco de procesos de aprendizaje colaborativo en línea.
}

La internacionalización en el aula en Licenciaturas en Educación Infantil en el Caribe colombiana: una perspectiva dacente Luisa F. Echeverría King, Fuensanta Hernández-Pinal, Diana Lagu de Vergara

DOI: http://dx.dai.org//0.22458/caes.vlliz.2831

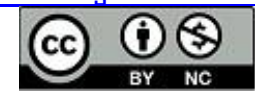


profesorado reportó acudir a juegos y estrategias como la clase invertida y laboratorios didácticos. Cabe resaltar que las innovaciones pedagógicas hacen parte de las estrategias planteadas por Cecar en el Proyecto Educativo del Programa (Cecar, 2019).

En cuanto a los métodos de evaluación se relacionan proyectos, evaluaciones formativas, exposiciones y autoevaluaciones; igualmente el cuerpo docente comunicó poseer rúbricas para guiar su proceso de evaluación.

Por último, se revisaron los recursos educativos utilizados por docentes de la Licenciatura en Pedagogía Infantil, estos reportaron usar videos, artículos especializados, blogs, chats, así como la plataforma Moodle y software educativos; estos recursos apoyan al programa a interactuar con contextos internacionales, tal y como lo expone el Proyecto Educativo Institucional de la IES (Cecar, 2017). En la tabla 4 se observan los resultados de manera sintetizada.

Las metodologías docentes utilizadas por el profesorado del programa de Educación Infantil de la Universidad de Guajira se tratan de comparaciones internacionales en las clases, clases espejo y videoconferencias, rincones didácticos, visitas a escuelas, clases magistrales, proyectos, así como debates y pedagogías activas. Cabe resaltar que las comparaciones internacionales le sirven a construcción o re-construcción de las diferentes áreas de estudio promovidas desde el Proyecto Educativo del Programa, especialmente desde metodologías horizontales que apoyen la interacción entre los docentes y estudiantes (Universidad de la Guajira, 2016).

La internacionalización en el aula en Licenciaturas en Educación Infantil en el Caribe colombiana: una perspectiva dacente Luisa F. Echeverría King, Fuensanta Hernández-Pinal, Diana Laga de Vergara

DDI: http://dx.dai.org/I0.22458/caes.vlli2.2831

(c) (i) $\$$

Artícula prategida por licencia Ereative Cammans 
Tabla 4. Acciones lideradas para la internacionalización del proceso enseñanza-aprendizaje en Cecar para la Licenciatura en Pedagogía Infantil

\begin{tabular}{|c|c|c|}
\hline Categorías & $\begin{array}{c}\text { Acciones lideradas por los } \\
\text { docentes }\end{array}$ & Extractos de las entrevistas \\
\hline 1. Metodologías docente & $\begin{array}{l}\text {-Debates } \\
\text {-Clases magistrales } \\
\text {-Clases espejo } \\
\text {-Pedagogías activas (Aprendizaje } \\
\text { basado en proyectos/problemas) } \\
\text {-Juegos } \\
\text {-Clase invertida } \\
\text {-Laboratorios }\end{array}$ & $\begin{array}{l}\text { "Estamos hablando de docentes que } \\
\text { son pedagogos, que tienen en sus } \\
\text { manos la educación infantil y como tal } \\
\text { ellos qué necesitan, saber, o sea, si yo } \\
\text { no juego, si yo no disfruto el juego, } \\
\text { cómo voy a pretender que mis } \\
\text { estudiantes jueguen y disfruten" } \\
\text { (Docente Cecar 3, Profesora de } \\
\text { asignatura de Psicomotricidad y } \\
\text { Juego). }\end{array}$ \\
\hline 2. Métodos de evaluación & $\begin{array}{l}\text {-Proyectos } \\
\text {-Evaluación formativa } \\
\text {-Exposiciones } \\
\text {-Rúbricas } \\
\text {-Autoevaluaciones }\end{array}$ & $\begin{array}{l}\text { "Pero para el segundo corte nuestra } \\
\text { estrategia de evaluación es la apuesta } \\
\text { por una propuesta para el desarrollo } \\
\text { de la equidad en la primera infancia" } \\
\text { (Docente Cecar 1, Profesora de } \\
\text { asignatura de Equidad de Género). }\end{array}$ \\
\hline 3. Recursos educativos & $\begin{array}{l}\text {-Videos } \\
\text {-Artículos especializados } \\
\text {-Blogs } \\
\text {-Chats } \\
\text {-Plataforma Moodle } \\
\text {-Softwares educativos }\end{array}$ & $\begin{array}{l}\text { "Bueno, son muchos, por ejemplo, los } \\
\text { links, los blogs y todos los juegos que } \\
\text { son en línea, que ellos pueden ir } \\
\text { desarrollando e implementando y me } \\
\text { parece que son fundamentales, la } \\
\text { plataforma Moodle" (Docente Cecar } 4 \text {, } \\
\text { Profesora de asignatura de Práctica). }\end{array}$ \\
\hline
\end{tabular}

Fuente: Elaboración propia con base a información recogida en entrevistas

En cuanto a los métodos de evaluación, se observan estrategias como lo son la autoevaluación, coevaluación y heteroevaluación, así como presentaciones, informe, talleres, exámenes escritos, proyectos y observaciones en escuelas. Los recursos educativos principalmente utilizados son aquellos mediados por las TIC, como lo son blogs, videos, bases de datos, bibliotecas virtuales, así como artículos especializados y bibliografía extranjera, como se observa en la tabla 5 .

La internacionalización en el aula en Licenciaturas en Educación Infantil en el Caribe colombiana: una perspectiva dacente Luisa F. Echeverría King, Fuensanta Hernández-Pinal, Diana Laga de Vergara

DOI: http://dx.dai.org/l0.22458/caes.vlliz.2831

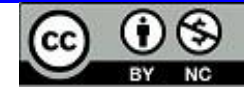


Tabla 5. Acciones lideradas para la internacionalización del proceso enseñanza-aprendizaje en la Uniguajira para la Licenciatura en Educación Infantil

\begin{tabular}{|c|c|c|}
\hline Categorías & $\begin{array}{c}\text { Acciones lideradas por los } \\
\text { docentes }\end{array}$ & Extractos de las entrevistas \\
\hline 1. Metodologías docente & $\begin{array}{l}\text {-Comparaciones internacionales } \\
\text {-Clases espejo y videoconferencias } \\
\text {-Rincones didácticos } \\
\text {-Visitas a escuelas } \\
\text {-Clases magistrales } \\
\text {-Proyectos } \\
\text {-Pedagogías activas } \\
\text {-Debates }\end{array}$ & $\begin{array}{l}\text { "Les hablo de políticas sociales, de } \\
\text { políticas públicas, de esos acuerdos y } \\
\text { alianzas estratégicas que se } \\
\text { establecen, de los programas que se } \\
\text { adelantan primero, desde el objetivo } \\
\text { del milenio, pasando por todos los } \\
\text { organismos por ejemplo UNICEF, lo } \\
\text { que es la ONU, la OEA" (Docente } \\
\text { Uniguajira 1, Profesora de asignatura } \\
\text { de Gestión de Proyectos Sociales). }\end{array}$ \\
\hline 2. Métodos de evaluación & $\begin{array}{l}\text {-Autoevaluación } \\
\text {-Coevaluación } \\
\text {-Heteroevaluación } \\
\text {-Presentaciones } \\
\text {-Informes } \\
\text {-Talleres } \\
\text {-Proyectos } \\
\text {-Observaciones en escuelas } \\
\text {-Exámenes escritos }\end{array}$ & $\begin{array}{l}\text { "Permanentemente es teoría y práctica } \\
\text { ese es mi sistema de evaluación } \\
\text { [...]Esto no es que se va a improvisar } \\
\text { en el momento, esto hay un sistema } \\
\text { que se llama el antes, durante y el } \\
\text { después, en el después que es cuando } \\
\text { nos vamos para la casa se le dejan las } \\
\text { actividades propuestas para el día } \\
\text { siguiente, el consultar y el hacer que es } \\
\text { una estrategia, algo dinámico, algo } \\
\text { lúdico traer un sombrero, traer un } \\
\text { muñeco, los materiales, cuando vemos } \\
\text { la teoría, dejamos proyectada la teoría } \\
\text { y hacemos la parte práctica" (Docente } \\
\text { Uniguajira } 10 \text {, Profesora de asignatura } \\
\text { de psicología y sexualidad infantil). }\end{array}$ \\
\hline 3. Recursos educativos & $\begin{array}{l}\text {-Blogs } \\
\text {-Recursos tecnológicos } \\
\text {-Videos } \\
\text {-Artículos especializados } \\
\text {-Bibliografía extranjera } \\
\text {-Bases de datos } \\
\text {-Bibliotecas virtuales }\end{array}$ & $\begin{array}{l}\text { "Las redes, porque el estudiante utiliza } \\
\text { mucho las redes para consultar, pero la } \\
\text { universidad cuenta creo que con } 2,3 \\
\text { plataformas especializadas en } \\
\text { biblioteca, y ellos a veces consiguen } \\
\text { cosas ahí pero muchas veces utilizan lo } \\
\text { más común, Google, y otras similares, } \\
\text { pero bueno a pesar de que ellos } \\
\text { cuentan con esas bibliotecas virtuales" } \\
\text { (Docente Uniguajira 7, Profesor de } \\
\text { asignatura de trabajo de grado 1). }\end{array}$ \\
\hline
\end{tabular}

Fuente: Elaboración propia con base a información recogida en entrevistas

La internacionalización en el aula en Licenciaturas en Educación Infantil en el Caribe colombiana: una perspectiva dacente Luisa F. Echeverría King, Fuensanta Hernández-Pinal, Diana Laga de Vergara

DOI: http://dx.dai.org/l0.22458/caes.vlliz.2831

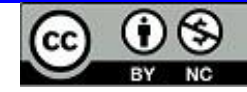


$\overline{\text { El último apartado de la entrevista buscó recoger sugerencias por parte de los docentes para }}$ apoyar la internacionalización del proceso enseñanza-aprendizaje. En el caso del personal docente de Cecar, este sugiere tener claridad hacia lo que se quiere y se planea frente a la internacionalización, definir objetivos de internacionalización en el aula, así como generar una pedagogía entre el equipo para apoyarse en este proceso. Esto se relaciona especialmente con la dimensión de "Internacionalización intencionada" del Consejo Europeo de Agencias de Acreditación (2017), dado que, si no se tienen objetivos y metas claras de internacionalización para el programa y estrategias para monitorear estas metas, es complicado poder alcanzarlas.

Asimismo proponen generar más oportunidades de intercambio con pares internacionales, impulsar innovaciones por medio de las TIC, fomentar alianzas internacionales para apoyar procesos de movilidad como por ejemplo, pasantías en colegios internacionales. Asimismo consideran que el talento humano debe fortalecerse y prepararse para la internacionalización por medio de capacitaciones y la dedicación de personal para este particular, así como consideran que requieren acompañamiento en el proceso, como se observa en la figura 3 :

La internacionalización en el aula en Licenciaturas en Educación Infantil en el Caribe colombiana: una perspectiva dacente Luisa F. Echeverría King, Fuensanta Hernández-Pinal, Diana Laga de Vergara

DQI: http://dx.dai.org/L10.2Z458/caes.vlliz.2831

(c) $\underset{\mathrm{BY}}{\mathrm{BY}} \mathrm{(a)}$ 
Figura 3. Sugerencias para la internacionalización del proceso enseñanza-aprendizaje en Cecar

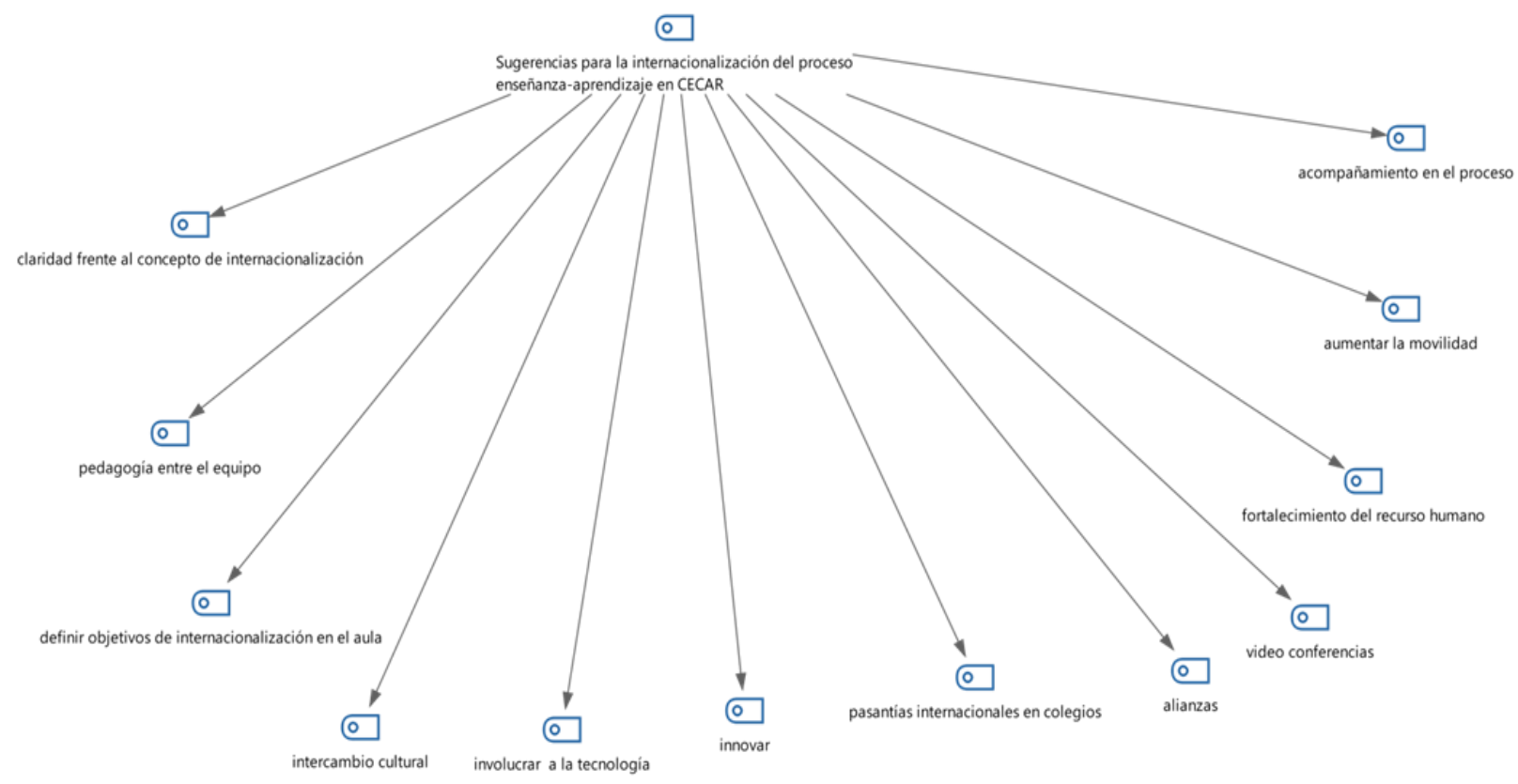

En el caso de la Universidad de la Guajira, el profesorado sugiere invitar a expertos de otros países para apoyar la internacionalización del proceso enseñanza-aprendizaje, así como incluir asignaturas internacionales en los programas académicos y la generación de foros en el aula con aliados extranjeros. Estas sugerencias van en la línea con lo propuesto por parte del Consejo Europeo de Agencias de Acreditación (2017), el cual expone en la dimensión "Enseñanza y Aprendizaje" que la internacionalización debe ser transversal al proceso enseñanza-aprendizaje, alineando el currículo, los objetivos de internacionalización del programa y los resultados de aprendizaje internacionales. Se recomienda en este sentido la organización de eventos académicos internacionales, así como el uso de las TIC para las

La internacionalización en el aula en Licenciaturas en Educación Infantil en el Caribe colambiana: una perspectiva dacente Luisa F. Echeverría King, Fuensanta Hernández-Pinal, Diana Lagu de Vergara

DDI: http://dx.dai.org//0.22458/caes.vlliz.2831

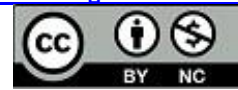

Artícula protegida par licencia Creative Cammans 
acciones formativas, el fomento de la movilidad y el intercambio entre pares internacionales y la capacitación del profesorado. Por último, se sugiere igualmente la multiplicación de conocimientos obtenidos en el exterior, recursos para apoyar el proceso, más divulgaciones de oportunidades internacionales y trabajos de grado con enfoque internacional.

Figura 4. Sugerencias para la internacionalización del proceso enseñanza-aprendizaje en Uniguajira

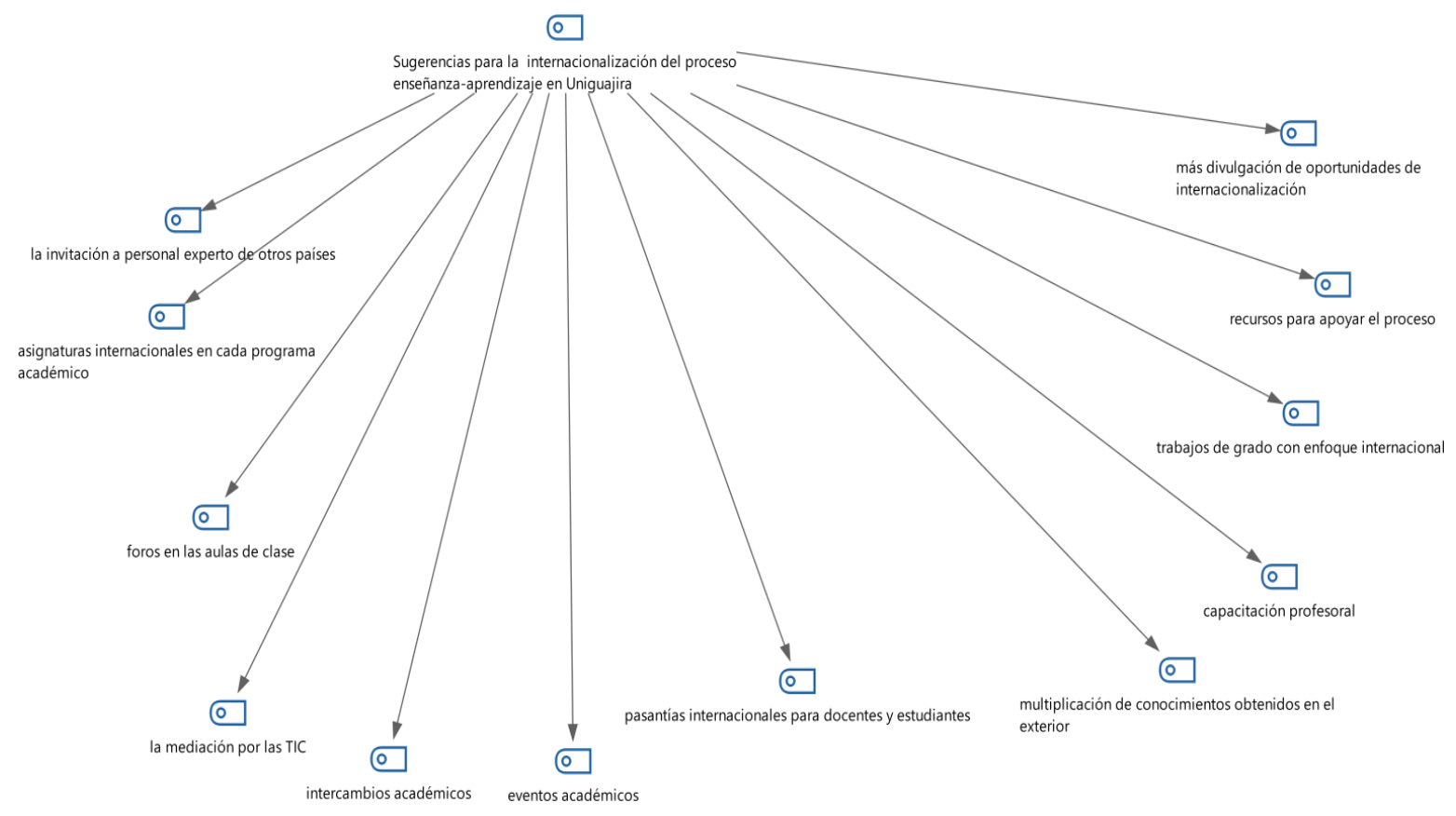

\section{Conclusiones}

La internacionalización en el aula como puede verse por medio de esta investigación, va de la mano con las proyecciones y direccionamientos institucionales y de los programas frente a la internacionalización, así como también se relaciona con el modelo pedagógico priorizado por cada IES. En este sentido se hace necesario contar con metas y objetivos claros para la

La internacionalización en el aula en Licenciaturas en Educación Infantil en el Caribe colombiana: una perspectiva dacente Luisa F. Echeverría King, Fuensanta Hernández-Pinal, Diana Lagu de Vergara

DDI: http://dx.dai.org//0.22458/caes.vlli2.2831

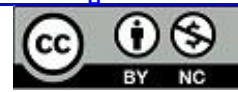

Artícula protegida par licencia Creative Cammans 
$\overline{\text { internacionalización de los programas académicos, así como de estrategias de seguimiento }}$ para monitorear el avance frente a estas metas.

En cuanto a los programas de Pedagogía y Educación Infantil, se observa la importancia de generar innovaciones curriculares por medio de la internacionalización, que fomenten el trabajo colaborativo con pares internacionales, el uso de las tecnologías de la información, las movilidades internacionales y las pedagogías activas. La internacionalización puede apoyar el desarrollo y mejoramiento de la calidad de los programas, sin embargo esta debe estar bien direccionada. Es importante mencionar que los programas de licenciatura en Colombia tienen el reto de formar a la nueva generación de maestros y en este sentido estos deben responder a las necesidades del entorno y de las sociedades, las cuales cada vez están más interconectadas como efecto de la globalización y la sociedad del conocimiento. Se hace necesario plantearse desde los programas académicos la generación de acciones específicas para las disciplinas de internacionalización curricular, más allá de las estrategias institucionales, para poder generarle al profesorado en formación oportunidades de intercambio y aprendizaje con pares internacionales.

Estas acciones sin embargo requieren de recurso humano capacitado y disponible para poder llevar a cabo estas actividades, así como infraestructura y recursos, por lo cual las instituciones deberán plantearse esquemas de formación de recurso humano que apoyen la inclusión de la internacionalización en el aula. En todo caso y teniendo en cuenta el modelo de aseguramiento de la calidad de programas en Colombia, así como las dimensiones para la calidad en la internacionalización del Consejo Europeo de Agencias de Acreditación, las IES deberán contar con estrategias coordinadas que le apunten al desarrollo de la calidad de los programas gracias a la inclusión de componentes internacionales e interculturales.

La internacionalización en el aula en Licenciaturas en Educación Infantil en el Caribe colombiana: una perspectiva dacente Luisa F. Echeverría King, Fuensanta Hernández-Pinal, Diana Lago de Vergara

DDI: http://dx.dai.org/I0.22458/caes.vlli2.2831

(c) (i) (8)

Artícula prategida par licencia Lreative Cammans 


\section{Referencias bibliográficas}

Arango, A., y Acuña, L., (2018). La internacionalización del currículo y su relación con las condiciones de calidad en los programas académicos de educación superior para la obtención de registro calificado. Revista ObIES, 2, 35-49. https://doi.org/10.14483/25905449.12739

Asociación Europea para la Calidad de la Educación Superior (2015). Criterios y Directrices para el Aseguramiento de Calidad en el Espacio Europeo de Educación Superior (ESG). https://cutt.ly/Lf6nE5K

Cecar (2017). Proyecto Educativo Institucional. https://cutt.ly/gf3Cs43

Cecar (2019). Proyecto Educativo del Programa de la Licenciatura en Pedagogía Infantil de Cecar. https://cutt.ly/ff5xbD3

Colás Bravo M., Buendía Eisman, L., y Hernández-Pina, F. (2009). Competencias científicas para la realización de una tesis doctoral. Guía metodológica de la elaboración y presentación. Editorial Da Vinci.

Consorcio Europeo de Agencias de Acreditación (2017). The Guide to Quality in Internationalisation. https://cutt.ly/Tf6nIG3

Creswell, J. (2012). Educational Research. Planning, Conducting and Evaluating Quantitative and Qualitative Research. Pearson Education Inc.

Crosling, G., Edwards, R. y Schroder, B. (2008). Internationalizing the curriculum: the implementation experience in a Faculty of Business and Economics. Journal of Higher Education Policy and Management, 30(2), 107-121. doi: 10.1080/13600800801938721.

Echeverría, L. y Lafont, T. (2018). Retos y potencialidades de la movilidad académica estudiantil internacional: Caso Nodo Caribe de la Red Colombiana para la Internacionalización de la Educación Superior (RCl). En M. Oregioni y A. Taborga (Coord.), Dinámicas de Internacionalización Universitaria en América Latina (pp. 157174). Grafikart.

La internacionalización en el aula en Licenciaturas en Educación Infantil en el Caribe colombiana: una perspectiva dacente Luisa F. Echeverría King, Fuensanta Hernández-Pinal, Diana Lagu de Vergara

DII: http://dx.dai.org/I0.22458/caes.vlliz.2831

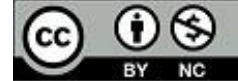


Departamento de la Guajira (1976, 12 de noviembre). Decreto 523 de 1976, por el cual se crea, organiza y reglamenta el funcionamiento de la Universidad Experimental de la Guajira. https://cutt.ly/ofZoj7N

De Wit, H. (2011). Globalización e internacionalización de la educación superior. Revista de Universidad y Sociedad del Conocimiento (RUSC), 8(2), 77-84. https://cutt.ly/1f6nGO9

De Zan, A., Paipa, L. y Parra, C. (2011). Las competencias: bases para la internacionalización de la educación superior. Revista Educación en Ingeniería, 11, 44-54. https://cutt.ly/kf6nK6o

Gamboa, A., Lago, D. y Hernández-Pina, F. (2016). Calidad de la Docencia Universitaria: Comprensión de los discursos y políticas institucionales de la universidad pública en Norte de Santander. Saber, Ciencia y Libertad, 11(1), 197-210. https://cutt.ly/df6nZmK

Hernández-Pina, F. y Maquilón Sánchez, J. (2015). El proceso de investigación científica. En F. Hernández-Pina, J. Maquilón Sánchez, J. Saéz de Tejada, T. Izquierdo Rus (Eds.), Investigación y análisis de datos para la realización de TFG, TFM y tesis doctoral (p. 11-34). Compobell.

Hoff, J. y Kappler, B. (2005). Integrating Intercultural Learning into Education Abroad Programming. En J. Brockington, W. Hoffa y P. Martin (Eds.), Nafsa's guide to education abroad. For advisers and administrators (pp. 195-206). Nafsa.

Leask, B. (2009). Using formal and informal curricula to improve interactions between home and international students. Journal of Studies in International Education, 13(2), 205221. doi: https://doi.org/10.1177/1028315308329786.

Leask, B. (2014). Internationalizing the Curriculum and all Students' Learning. International Higher Education, 78, 5-6. https://doi.org/10.6017/ihe.2014.78.5798

Mayring, P. (2002). Einführung in die Qualitative Forschung. Beltz Verlag.

Ministerio de Educación Nacional (2019). Decreto 1330 de 2019, por el cual se sustituye el Capítulo 2 y se suprime el Capítulo 7 del Título 3 de la Parte 5 del Libro 2 del Decreto 1075 de 2015 Único Reglamentario del Sector Educación.

Peña, M. y Aponte, C. (2018). Internacionalización Conectiva. El currículo en un mundo en red. Dirección de Publicaciones de la Universidad Católica de Santiago de Guayaquil.

La internacionalización en el aula en Licenciaturas en Educación Infantil en el Caribe colombiana: una perspectiva dacente Luisa F. Echeverría King, Fuensanta Hernández-Pinal, Diana Laga de Vergara

DDI: http://dx.dai.org//0.22458/caes.vlli2.2831

$$
\text { (c) (i) (5) }
$$


Peña, M. (2019). Internacionalización conectiva del currículo. Fundamentos epistemológicos y metodológicos. Revista Espacios, 40(26), 2-9. https://cutt.ly/rf6n5eZ

Sawir, E. (2013). Internationalisation of higher education curriculum: the contribution of international students. Globalisation, Societies and Education, 11(3), 359-378. doi: http://dx.doi.org/10.1080/14767724.2012.750477

Universidad de la Guajira (2016). Proyecto Educativo del Programa Licenciatura en Educación Infantil. https://cutt.ly/9gq1FKe

Universidad de la Guajira (2017). Proyecto Educativo Institucional 2017-2030. https://cutt.ly/wf5cDS1 